\title{
Efecto de la administración de líquido ruminal fresco sobre algunos parámetros productivos en ovinos criollos
}

\section{Effect on weight gain, rumen bioactivity and rumen pH in weaned sheep using fresh rumen fluid from cattle}

\author{
Carlos Rodríguez M, ${ }^{1 *}$ Esp, Angela Rodríguez S, ${ }^{1} \mathrm{MVZ}$.
}

\begin{abstract}
${ }^{1}$ Universidad Pedagógica y Tecnológica de Colombia, Facultad de Ciencias Agropecuarias, Grupo de Investigación en Bioquímica y Nutrición Animal - GIBNA. Tunja, Colombia. *Correspondencia: ceromol@gmail.com
\end{abstract}

Recibido: Enero de 2010; Aceptado: Abril de 2011.

\section{RESUMEN}

Objetivo. Evaluar el efecto de la administración de líquido ruminal fresco (LRF) de bovino sobre la ganancia de peso, bioactividad ruminal y $\mathrm{pH}$ ruminal en ovinos. Materiales y métodos. Se utilizaron nueve ovinos destetados con edades y pesos homogéneos, distribuidos de forma aleatoria en tres grupos, a los cuales se les suministró tres tratamientos diferentes: tratamiento 1, $400 \mathrm{ml}$ de LRF; tratamiento 2, $200 \mathrm{ml}$ de LRF y tratamiento 3 como control (sin LRF). El LRF se administro vía oral, usando una dosis única al inicio del estudio. Para la evaluación de los parámetros, se inició con una medición el día 0 y luego se realizaron mediciones cada $15 \mathrm{~d}$ durante un periodo de $60 \mathrm{~d}$. Para el análisis estadístico se realizó ANOVA y las diferencias se comprobaron mediante el test de comparación de medias de Tukey. Resultados. El promedio de la ganancia de peso diario, fue de 194.4 y $169.4 \mathrm{~g} / \mathrm{d}$ en los animales tratados con 400 y $200 \mathrm{ml}$ de $L R F$, respectivamente y de $157.8 \mathrm{~g} / \mathrm{d}$ en los animales que no recibieron LRF, siendo estas diferencias estadísticamente no significativas $(p>0.05)$. La bioactividad ruminal presentó cambios significativos tras la administración de $400 \mathrm{ml}$ de LRF $(p<0.05)$. El pH solo mostró cambios significativos en la medición realizada a los 15 d postadministración de $400 \mathrm{ml}$ de LRF. Conclusiones. La administración de LRF no provocó aumentos significativos sobre la ganancia diaria de peso, no obstante, mostró efectos favorables sobre la bioactividad ruminal.

Palabras clave: Ganancia de peso, pH, ruminal, tratamientos, ovinos (Fuente: CAB). 


\section{ABSTRACT}

Objetive. To determine the effect on weight gain, rumen bioactivity and rumen $\mathrm{pH}$ in weaned sheep using fresh rumen fluid (FRF) from cattle. Materials and methods. Nine weaned sheep with (aproximated same age and weight), were randomized into three groups: Treatment 1), $400 \mathrm{ml}$ of FRF, Treatment 2), $200 \mathrm{ml}$ of FRF and Treatment 3), and 4) Control without FRF. The FRF was administered through oral route, using a single dose as baseline. Parameters studied, were taking at day 0 and then every 15 days during a 60 days period. For statistical analysis ANOVA and the Tukey'stest for each mean comparison were performed. Results. There were not significant differences ( $p>0.05)$ for daily weight gain, average profits between treated groups and control group $(194.4 \mathrm{~g} / \mathrm{d}, 169.4 \mathrm{~g} / \mathrm{d}$ and $157.8 \mathrm{~g} / \mathrm{d}$ respectively). Rumen bioactivity does showed changes after the administration of $400 \mathrm{ml}$ of FRF $(p<0.05)$. The $\mathrm{pH}$ showed only significant changes after 15 days of the administration of $400 \mathrm{ml}$ of FRF. Conclusions. The administration of FRF did not cause significant increases on daily weight profit; nevertheless, it showed favorable effects on rumen bioactivity in weaned sheep.

Key words. Weight gain, $\mathrm{pH}$, rumen, treatments, sheep (Source: $C A B$ ).

\section{INTRODUCCIÓN}

Los rumiantes, principalmente en el periodo comprendido entre el nacimiento y el destete, sufren un cambio drástico de las condiciones del sistema digestivo $(1,2)$, es por esto, que durante esta fase se debe tener un manejo adecuado de la alimentación, pues del correcto desarrollo adaptativo del rumiante dependerá la eficiencia en la conversión alimenticia y por ende favorecerá el crecimiento y desarrollo del animal (3). Los ovinos, al igual que el resto de los rumiantes utilizan su masiva comunidad de microorganismos, principalmente poblaciones mixtas interdependientes de bacterias y protozoos (4-6), para generar a partir de la fermentación ruminal tanto la energía como la proteína que tiene disponible, hecho que evidencia la importancia de la manipulación de estos procesos con el fin de obtener una significativa eficiencia en la utilización de nutrientes para una mayor producción animal (7).

En respuesta a esta problemática y aprovechado el hecho de que el contenido ruminal posee gran cantidad de flora y fauna microbiana y productos de la fermentación ruminal (4), este producto, se ha usado por décadas como alternativa en la alimentación de rumiantes (8), pollos $(9,10)$, cerdos de engorde $(11)$ y en general para la elaboración de bloques nutricionales para la alimentación animal (12), gracias a sus características químicas, biológicas, bromatológicas, microbiológicas, por su amplia disponibilidad y por no competir directamente con la alimentación humana (8).

La fase liquida del contenido ruminal, conocido como líquido ruminal cuenta con una población alta de microorganismos, encargada de la digestión de los nutrientes de los alimentos que son ingeridos por los animales. En el LRF, aproximadamente un $4 \%$ del volumen total corresponde a volumen microbiano, donde las bacterias constituyen la mitad de la biomasa en el rumen normal y son responsables de la actividad metabólica; de igual forma, se encuentran hongos, que constituyen hasta el $8 \%$ de la biomasa intra ruminal y se ubican en la ingesta de lento movimiento, evitando su rápido lavado y contribuyen a la digestión de forrajes de baja calidad. Por otra parte, los protozoos son los organismos más notables en el rumen, forman gran proporción de la biomasa, 
entre un $20-40 \%$, pero su contribución es menor por la gran retención y la menor actividad metabólica (4).

El objetivo de este estudio fue evaluar la respuesta productiva y fisiológica de ovinos tras la administración LRF, obtenido del rumen de bovinos sanos, esperando con esto que al aumentar la población de microorganismos en el rumen de los ovinos, exista una mayor eficiencia en la digestión de los nutrientes y por ende una mayor productividad.

\section{MATERIALES Y MÉTODOS}

Sitio de estudio. El trabajo fue desarrollado en la granja experimental Tunguavita de la Universidad Pedagógica y Tecnología de Colombia (UPTC), ubicada en el municipio de Paipa, departamento de Boyacá, a una altitud de 2590 msnm, latitud $05^{\circ} 45^{\prime}$ norte, longitud $73^{\circ} 45^{\prime}$ oeste, con una temperatura promedio de $14.3^{\circ} \mathrm{C}$, humedad relativa del $78 \%$ y precipitación promedio de $705.9 \mathrm{~mm}^{3} /$ año.

Animales. Se utilizaron nueve ovinos destetados y con pesos iniciales promedio de $20 \pm 2 \mathrm{~kg}$. Los animales fueron mantenidos en pastoreo rotacional a base de Pennisetum clandestinum (kikuyo) y Trifolium pratense (trébol rojo) y con suministro constante de agua limpia.

Ocho días antes del inicio del estudio los animales se desparasitaron con albendazol al $10 \%$, según la dosis recomendada para la especie (13) y se les administró una dosis única de suplementos vitamínicos orales, $(A, D$ y $E)$, según recomendaciones de algunos autores (14).

Los ovinos fueron distribuidos al azar mediante un diseño completamente aleatorio en tres tratamientos con LRF, tratamiento 1, $400 \mathrm{ml}$ de LRF; tratamiento 2, $200 \mathrm{ml}$ de LRF y un tercer tratamiento, tomado como control absoluto, en el cual los animales no recibieron tratamiento con LRF, sin embargo se les administró vía oral $200 \mathrm{ml}$ de agua potable, con el fin de simular las mismas condiciones de estrés en todos los animales. De otro lado el motivo por el cual solo se utilizaron tres animales por grupo fue por cuestiones administrativas y éticas de la granja Tunguavita, debido a que no se conocía la reacción fisiológica de los animales ante estos tratamientos y por ende no se quería causar daño a los animales en estudio.

Obtención y administración del LRF. El LRF se recolectó de un donante bovino adulto, sano, utilizando la técnica quirúrgica "ruminotomía" (15). Se colectó inicialmente contenido ruminal, que fue filtrado y tamizado hasta obtener finalmente la cantidad necesaria de LRF, sobre el que se determinó bioactividad y $\mathrm{pH}$ ruminal.

El LRF, se administró vía oral, usando una dosis única al inicio del experimento, en el día 0; pues para algunos autores una sola administración de LRF es suficiente para obtener buenos resultados en ganancias de peso, mejorando algunos parámetros fisiológicos como, bioactividad y $\mathrm{pH}$ ruminal (16).

Parámetros evaluados. Se realizaron mediciones de la ganancia de peso y bioactividad y $\mathrm{pH}$ ruminal. Estos parámetros fueron evaluados antes de la administración del LRF (día 0) y luego, con una frecuencia de $15 \mathrm{~d}$, por un tiempo experimental de 60 d. La medición de los parámetros se realizó bajo las mismas condiciones para evitar variaciones en los resultados, manejando siempre la misma hora para los muestreos.

Pesaje de los animales. Los animales fueron pesados cada 15 dias en una balanza electrónica. A través de estos pesos, se estimó la ganancia diaria de peso, determinada cada quince días y promediada al final del estudio, de igual modo se determinó la ganancia de peso al final del estudio.

Bioactividad del líquido ruminal. Para la evaluación de la bioactividad ruminal se utilizó el test de tiempo de reducción del azul de metileno (17), para tal fin se tomó una muestra de $10 \mathrm{ml}$ de líquido ruminal por ruminocentesis en el flanco izquierdo. La muestra de líquido ruminal se depositó 
en un tubo de ensayo y luego se le adicionó una parte de azul de metileno al $0.03 \%$ a 20 partes de fluido extraído del rumen. En un segundo tubo, se colocó fluido ruminal, sin azul de metileno, el cual sirvió como control. Una vez expuesta la muestra de líquido ruminal al azul de metileno, se contabilizó el tiempo, en minutos, que tardó en presentarse decoloración del reactivo.

Análisis del pH del líquido ruminal. Para la medición del $\mathrm{pH}$, se tomó $10 \mathrm{ml}$ de líquido ruminal, por medio de ruminocentesis, en donde se introdujo un $\mathrm{pH}$-metro que determinó la acidez del LRF (18).

Análisis estadístico. Se realizó un diseño completamente al azar, un análisis de varianza y un test de comparación de medias de Tukey, con el uso del programa SPSS para Windows, versión 11.5.1 del 2002.

\section{RESULTADOS}

Ganancia diaria de peso (g/d). LoS resultados de las ganancias de peso diarias obtenidas de los pesajes realizados cada quince días y el promedio de ganancia de peso diario durante el estudio, se reportan en la tabla 1 . En cuanto a la ganancia diaria de peso, no se observan diferencias significativas $(p>0.05)$, hecho que se observó en la totalidad de los resultados obtenidos durante el estudio. De igual modo en la ganancia de peso diaria promediada, no existieron diferencias $(p>0.05)$.

Tabla 1. Ganancia de peso diaria (g/día).

\begin{tabular}{lccccc}
\hline \multirow{2}{*}{ Tratamiento } & \multicolumn{4}{c}{ Quincena } & $\begin{array}{c}\text { Promedio } \\
\text { al final } \\
\text { tratamiento }\end{array}$ \\
\cline { 2 - 6 } & $\mathbf{1}$ & $\mathbf{2}$ & $\mathbf{3}$ & $\mathbf{4}$ & $194.4^{\mathrm{a}}$ \\
\hline 400ml LRF & $255.6^{\mathrm{a}}$ & $155.6^{\mathrm{a}}$ & $177.8^{\mathrm{a}}$ & $188.9^{\mathrm{a}}$ & $169.4^{\mathrm{a}}$ \\
$200 \mathrm{ml} \mathrm{LRF}$ & $200^{\mathrm{a}}$ & $166.7^{\mathrm{a}}$ & $155.6^{\mathrm{a}}$ & $155.6^{\mathrm{a}}$ & $157.8^{\mathrm{a}}$ \\
$\begin{array}{l}\text { Control } \\
\text { (Oml LRF) }\end{array}$ & $188.9^{\mathrm{a}}$ & $155.6^{\mathrm{a}}$ & $133.3^{\mathrm{a}}$ & $153.3^{\mathrm{a}}$ & 150 \\
\hline
\end{tabular}

Medias con letras diferentes en la misma columna, difieren estadísticamente $(p<0.05)$.

Ganancia de peso final (g). LoS resultados se reportan en la tabla 2 . Con respecto a la ganancia de peso al final del estudio no se observó diferencias significativas $(p>0.05)$ entre los diferentes tratamientos.
Tabla 2. Ganancia de peso final.

\begin{tabular}{cc}
\hline Tratamiento & $\begin{array}{c}\text { Ganancia de peso al final del } \\
\text { tratamiento }(\mathbf{k g})\end{array}$ \\
\hline $400 \mathrm{ml}$ de LRF & $11.6^{\mathrm{a}}$ \\
$200 \mathrm{ml}$ de LRF & $10.1^{\mathrm{a}}$ \\
Control (Oml de LRF) & $9.6^{\mathrm{a}}$ \\
\hline
\end{tabular}

Medias con letras diferentes en la misma columna, difieren estadísticamente $(p<0.05)$.

Bioactividad del líquido ruminal (min). Los resultados se observan en la tabla 3. La bioactividad reportada antes de la administración del LRF (día 0 ) en los grupos tratados, no mostraron diferencias ( $p>0.05)$. A los 15 días pos-administración, se observó efecto sobre la bioactividad ruminal para el tratamiento de $400 \mathrm{ml}$ de LRF, observando diferencia entre este tratamiento y el control $(p<0.05)$; este mismo comportamiento se observó para el día 45.

Tabla 3. Bioactividad del líquido ruminal (min).

\begin{tabular}{ccccccc}
\hline Tratamiento & \multicolumn{5}{c}{ Día } & $\begin{array}{c}\text { Promedio } \\
\text { durante el } \\
\text { estudio }\end{array}$ \\
\cline { 2 - 7 } & $\mathbf{0}$ & $\mathbf{1 5}$ & $\mathbf{3 0}$ & $\mathbf{4 5}$ & $\mathbf{6 0}$ & $5.4^{\mathrm{a}}$ \\
\hline $400 \mathrm{ml} \mathrm{LRF}$ & $7.7^{\mathrm{a}}$ & $5.3^{\mathrm{a}}$ & $5.3^{\mathrm{a}}$ & $5.3^{\mathrm{a}}$ & $5.5^{\mathrm{a}}$ & $6.0^{\mathrm{b}}$ \\
$200 \mathrm{ml} \mathrm{LRF}$ & $8.0^{\mathrm{a}}$ & $5.7^{\mathrm{ab}}$ & $6.0^{\mathrm{b}}$ & $6.3^{\mathrm{ab}}$ & $6.0^{\mathrm{a}}$ & $7.4^{\mathrm{c}}$ \\
$\begin{array}{c}\text { Control } \\
(\text { Oml LRF })\end{array}$ & $7.7^{\mathrm{a}}$ & $7.2^{\mathrm{b}}$ & $7^{\mathrm{c}}$ & $7.3^{\mathrm{b}}$ & $8^{\mathrm{b}}$ & \\
\hline
\end{tabular}

Medias con letras diferentes en la misma columna, difieren estadísticamente $(p<0.05)$.

Se encontraron diferencias $(p<0.05)$, para el análisis del día 30 entre todos los tratamientos. Para el día 60 , no se observaron diferencias entre los animales tratados con 400 y $200 \mathrm{ml}$ de LRF ( $p>0.05$ ) pero sí entre estos y el tratamiento control $(p<0.05)$. Finalmente, al promediar los resultados de la bioactividad ruminal durante los 60 días de estudio luego de la administración de LRF, se observó una mayor actividad ruminal en los animales tratados con $400 \mathrm{ml}$ de LRF $(p<0.05)$ con respecto a los otros tratamientos.

pH ruminal. En cuanto a los resultados de $\mathrm{pH}$ ruminal (Tabla 4 ), solo se observó un aumento significativo $(p<0.05)$ a los 15 días post-administración de LRF, en los animales tratados con 400 y $200 \mathrm{ml}$ de LRF frente a los animales del grupo control. 
Tabla 4. $\mathrm{pH}$ del liquido ruminal.

\begin{tabular}{|c|c|c|c|c|c|c|}
\hline \multirow{2}{*}{ Tratamiento } & \multicolumn{5}{|c|}{ Día } & \multirow{2}{*}{$\begin{array}{l}\text { Promedio } \\
\text { durante } \\
\text { el estudio }\end{array}$} \\
\hline & $\mathbf{0}$ & 15 & 30 & 45 & 60 & \\
\hline $400 \mathrm{ml}$ LRF & $5.4^{\mathrm{a}}$ & $6^{b}$ & $5.8^{\mathrm{a}}$ & $5.8^{\mathrm{a}}$ & $5.9^{a}$ & $5.9^{a}$ \\
\hline $300 \mathrm{ml}$ LRF & $5.3^{\mathrm{a}}$ & $5.9^{b}$ & $5.9^{a}$ & $5.6^{a}$ & $5.6^{\mathrm{a}}$ & $5.8^{\mathrm{a}}$ \\
\hline $\begin{array}{l}\text { Control, } \\
\text { Oml LRF }\end{array}$ & $5.4^{\mathrm{a}}$ & $5.4^{\mathrm{a}}$ & $5.5^{\mathrm{a}}$ & $5.5^{\mathrm{a}}$ & $56^{a}$ & $5.5^{\mathrm{a}}$ \\
\hline
\end{tabular}

\section{DISCUSIÓN}

Ganancia de peso al final del estudio. Con respecto a la ganancia de peso final observada (Tabla 2), se evidencia que los animales tratados con $400 \mathrm{ml}$ de LRF, ganaron 1.5 y $2.0 \mathrm{~kg}$ más de peso, con respecto a los animales tratados con $200 \mathrm{ml}$ y los animales del grupo control, respectivamente. No obstante, estas cifras no presentaron diferencias significativas ( $p>0.05$ ) entre los diferentes tratamientos. En investigaciones realizadas por Cuesta et al (16), luego de la administración de LRF, en una dosis única de $500 \mathrm{ml}$, encontraron una diferencia de peso de $3.5 \mathrm{~kg}$ entre los animales tratados con LRF vs los no tratados, esta diferencia de peso resultó ser significativa $(p<0.05)$, justificando plenamente la factibilidad económica del procedimiento aplicado.

Ganancia diaria de peso. Mediante el uso de diferentes cantidades de LRF se alcanzaron ganancias de peso, para la primera quincena de estudio de 255.6 y de $200 \mathrm{~g} / \mathrm{d}$ en los animales tratados con 400 y $200 \mathrm{ml}$ de LRF, respectivamente y de $188.9 \mathrm{~g} / \mathrm{d}$ en los animales del tratamiento control. En promedio al final del estudio, las ganancias diarias de peso fueron de 194.4 y $169.4 \mathrm{~g}$ en los animales tratados con 400 y $200 \mathrm{ml}$ de LRF, respectivamente y de $157.5 \mathrm{~g} / \mathrm{d}$ en los animales del tratamiento control, no siendo estas cifras significativas $(p>0.05)$. Aunque estas cifras son altas, no se observó un efecto positivo de la aplicación LRF, sobre la ganancia de peso, a diferencia de los resultados alcanzados por Cuesta et al (16), quienes luego de la administración de LRF en ovinos, reportó un efecto positivo luego de la aplicación $(p<0.05)$, alcanzando una ganancia diaria de peso de $143 \mathrm{~g} / \mathrm{d}$ y para el grupo testigo (manejado sin administración de LRF), ganancias diarias de peso de $27 \mathrm{~g} / \mathrm{d}$.

Cabrera et al (19), tras la suplementación de ovinos a base de sorgo y maíz, lograron ganancias de peso diarias de $270 \mathrm{~g} / \mathrm{d}$; Martínez et al (20), obtuvieron ganancias de peso de $244 \mathrm{~g} / \mathrm{d}$, luego de la suplementación a base de cascarilla de cerveza y Lara et al (21), con una dieta a base de grano entero de maíz y pasta de soya, reportaron una ganancia diaria de peso de $330 \mathrm{~g}$. Aunque estos trabajos no coinciden con la metodología empleada en el presente estudio, se observó que a pesar de no haberse usado ningún tipo de suplementación en los animales tratados con distintas dosis de LRF, las ganancias diarias de peso alcanzadas fueron similares a las obtenidas con la suplementación con diferentes fuentes de nutrientes.

Álvarez et al (22) tras la suplementación con tres tratamientos ( $\mathrm{T}$ ) distintos; $\mathrm{T} 1,30 \%$ de fruto de Entorolobium cyclocarpum; T2, 30\% de pollinaza y T3, $15 \%$ de Entorolobium cyclocarpum y $15 \%$ de pollinaza, lograron ganancias diarias de peso de 86, 74 y $111.7 \mathrm{~g}$, respectivamente. Con lo anterior, se puede concluir que a pesar de que los animales manejados en este estudio solo recibieron como tratamiento una dosis de LRF, se lograron ganancias de peso superiores a las reportadas en estas investigaciones, indicando que el manejo de las pasturas kikuyo - trébol rojo (Pennisetum clandestynum - Trifolium pratense) en la Granja Tunguavita de la UPTC, es apropiado.

Bioactividad del líquido ruminal. Luego de las pruebas de bioactividad del liquido ruminal, se observó que antes de la administración de LRF (día 0), este líquido mostró una bioactividad superior a los 7.5 min (Tabla 3), lo cual indica una actividad ruminal baja. Según lo reportado por Roussel (17), una decoloración del azul de metileno en muestras de LRF por encima de los 6 min es indicativo de una la flora ruminal menos activa, en cambio, una decoloración del reactivo inferior a 6 min indica la existencia de una flora ruminal activa. 
Para el muestreo realizado el día 15 postadministración de LRF, se observó en los animales tratados con 400 y $200 \mathrm{ml}$ de LRF, un tiempo de decoloración del azul de metileno inferior a los $6 \mathrm{~min}$, lo que indica la existencia de un flora ruminal activa $(16,17,23)$. Se reporta así, un efecto favorable de la administración de LRF $(p<0.05)$, para el grupo tratado con $400 \mathrm{ml}$ de LRF en el muestreo del día 15. Finalmente, al promediar los resultados de la bioactividad ruminal se observó la eficacia de la administración de LRF en los animales tratados con $400 \mathrm{ml}$ de LRF $(p<0.05)$, en donde la reducción del azul de metileno fue inferior a los $6 \mathrm{~min}$.

Los datos alcanzados en este estudio coinciden con los reportados por Cuesta et al (16), quienes alcanzaron tiempos de bioactividad ruminal de 5 min luego de la administración de $500 \mathrm{ml}$ de LRF en ovinos, encontrando a la vez, que los animales que no recibieron tratamiento con LRF, mantuvieron un tiempo de reducción de azul de metileno de $9 \mathrm{~min}$, lo que indica una baja bioactividad de la flora ruminal.

Consecuentemente, el mayor incremento de peso corporal y los mejores parámetros de bioactividad ruminal, se presentaron en los ovinos tratados con $400 \mathrm{ml}$ de LRF, principalmente si se comparan con los resultados alcanzados con el grupo control, estas ventajas son explicables por las consecuencias favorables que tiene el aumento de microorganismos ruminales luego de la transfaunación en la fermentación o mayor actividad de los microorganismos del rumen, coincidiendo con lo planteado por otros autores $(16,17,24,25)$. También coinciden con García y Cuesta (26) quienes encontraron correlación entre la bioactividad del líquido ruminal y la salud-producción del ganado bovino.

pH ruminal. Con respecto al efecto del LRF, sobre el $\mathrm{pH}$ ruminal se reportaron en la mayoría de los casos $\mathrm{pH}$ inferiores a 6; lo cual es denominado por algunos autores como acidosis ruminal subclínica (5.5 5.9), una alteración difícil de diagnosticar (27). Sin embargo, los animales tratados con $400 \mathrm{ml}$ de LRF, mostraron pH más altos, manteniéndose cercanos a un $\mathrm{pH}$ de 6 . Aunque estos resultados no lo diferencian con los de animales tratados con $200 \mathrm{ml}$ de LRF y los que no recibieron LRF ( $p>0.05)$, excepto, en el análisis realizado en el día 15 , donde estadísticamente, se observó un efecto favorable en el aumento del $\mathrm{pH}$ en los animales tratados con 400 y $200 \mathrm{ml}$ de $\operatorname{LRF}(p<0.05)$.

Montejo et al (28), $\mathrm{pH}$ inferiores a 6, provocan que las bacterias celulolíticas desciendan en número y que los protozoarios prácticamente desaparezcan. Esta condición puede repercutir sobre el potencial productivo del rebaño, al reducir la eficiencia de conversión alimenticia, debido a que la flora bacteriana mixta es la encargada de desdoblar y facilitar el metabolismo de los alimentos que el animal ingiere (29). A medida que el $\mathrm{pH}$ desciende, comienza la proliferación de bacterias acidófilas que provocan un descenso mas severo y peligroso del $\mathrm{pH}$; por ejemplo, $\mathrm{pH}$ inferiores a 4.7 crean condiciones ideales para el desarrollo del lactobacilo acidófilo con lo cual se produce más ácido.

En conclusión, con el uso de la técnica de transfaunación de LRF, no se logró un aumento de peso diario significativo $(p>0.05)$ en los animales tratados con 400 y $200 \mathrm{ml}$ de LRF comparándolo con los animales del grupo control que no recibieron LRF. Observándose este mismo efecto sobre el pH ruminal. No obstante, en cuanto a la bioactividad ruminal; los animales a los que se les suministró como dosis $400 \mathrm{ml}$ de LRF, mostraron estadísticamente una mayor actividad ruminal, arrojando lecturas de reducción de azul de metileno en un tiempo menor a $5.5 \mathrm{~min}$, durante todo el periodo de estudio, lo que se traduce en una mayor cantidad y por ende mayor actividad de la flora ruminal, con lo que se mejoran los índices fermentación, degradación y aprovechamiento de los nutrientes por parte del rumiante.

Para la primera medición de parámetros, realizada el día 15 post-administración del LRF, se observó que los animales tratados 
con $400 \mathrm{ml}$ de LRF, mostraron los mejores resultados en cuanto a ganancia de peso, bioactividad ruminal y $\mathrm{pH}$ ruminal, esto puede ser debido a que la población ruminal se encontraba en mayor cantidad gracias a que la administración del LRF era más reciente.

En cuanto a la dosis de LRF, se observó que con el uso de una única dosis se logra mantener en equilibrio la flora ruminal, pues durante el tiempo de estudio, la ganancia de peso diaria, bioactividad y $\mathrm{pH}$ ruminal, se mantuvieron constantes en los animales tratados, observándose cambios mínimos.

Teniendo en cuenta que algunos parámetros fueron mejorados con la administración de $400 \mathrm{ml}$ de LRF, se deben realizar investigaciones en donde se utilicen dosis mas altas de LRF de bovino, usando a la vez, varias aplicaciones para observar si con esta práctica se logra alcanzar mejores resultados. Además se recomienda identificar las bacterias y sus cantidades, luego de realizado un proceso de administración de LRF, para determinar cuantitativamente si las poblaciones bacterianas aumentan luego de realizado un proceso de transfaunación. Finalmente, para próximas investigaciones se podría trabajar grupos de animales con 6 o mas individuos, teniendo en cuenta que se demostró que este tipo de tratamientos no causa ninguna alteración en el bienestar de los animales.

\section{Agradecimientos}

A la Dirección de Investigaciones (DIN) y al Centro de Investigación de la Facultad de Ciencias Agropecuarias (INIAG) de la UPTC por el estudio, aprobación y financiamiento de este trabajo. A la Granja experimental Tunguavita de la UPTC, por el préstamo de los animales y las instalaciones necesarias para el desarrollo del proyecto.

\section{REFERENCIAS}

1. Díaz R, Laurencio $M$, Pérez $M$. Factores que influyen en el desarrollo ruminal de terneros de 0 a 6 meses de edad. [Trabajo de investigación]. Cuba: Universidad de Matanzas Camilo Cienfuegos. Facultad de Agronomía; 2008.

2. Belanche A, De La Fuente G, Ruíz Y, Calleja L, Balcells J. Desarrollo anatómico y microbiológico del rumen: efecto de la edad y tipo de dieta. Departamento de Producción Animal y Ciencia de los Alimentos. Universidad de Zaragoza (En línea) 2005 Febrero (Fecha de acceso 30 de Noviembre 2005); URL disponible en: http://www.aida-itea.org/jornada38/ nutricion/fisiologia_metabolismo/rfm-2 belanche.pdf.
3. Díaz R, Galindo J, Bocourt R, Laurencio $M$, Pérez $M$. Los microorganismos del rumen y su papel en la fisiología digestiva del rumiante. [Tesis Doctoral] Cuba: Universidad de Matanzas Camilo Cienfuegos, Facultad de Agronomía; 2008.

4. Araujo FO, Vergara LJ. Propiedades físicas y químicas del rumen. Arch Latinoam Prod Anim 2007; 15(Supl 1):34-38.

5. Robert AA, Barry F. Digestión en el estomago de los rumiantes. En: Swenson M, Reece WO. Fisiología de los animales domésticos de Dukes Tomo I. 2a Ed. México: Limusa-Wiley; 2001. 
6. Rotger CA. Fermentación ruminal degradación proteica y sincronización energía-proteína en terneras en cebo intenso. [Tesis Doctoral]. Barcelona: Universidad Autónoma de Barcelona, Facultad de Ciencia Animal; 2004.

7. Jaramillo LE, Espartaco MV, Hernández J, Pérez CF. Efecto de la raza e índice de temperatura sobre el comportamiento productivo de corderos del destete hasta los $35 \mathrm{~kg}$. Asociación Mexicana de Médicos Veterinarios Especialistas en Bovinos A. C. (En línea) 2006 agosto (Fecha de acceso 25 de noviembre de 2009); URL disponible en: http://ammveb. net/XXX\%20 CNB/memorias $\% 20$ 2006/pequenos_rumiantes/carteles/ rpeq03.htm.

8. Ruiz ZF, Hernández RE, Peña OJR, Gutiérrez $\mathrm{BH}$, Fuentes RJM. Efecto de la melaza mezclada con contenido ruminal de bovino alimantando a ovejas pelibuey. Asociación Mexicana de Médicos Veterinarios Especialistas en Bovinos A. C. (En línea) 2003 Junio (fecha de acceso 26 de abril de 2010); URL disponible en: http:// ammveb.net/XXVII\%20CNB/memorias/ Nutricion/Cartel/htm/Trabajo_72_Efecto_ de_la_melaza_mezclada_con.htm.

9. Bravo AM, Novoa B, Jabib L. Uso del contenido ruminal como parte de la dieta para pollos de engorde (Resumen). Rev MVZ Córdoba 2000; 5(2):30.

10. Otero PJ, Solano MA, Jabib RL, Oviedo L. Nivel óptimo de reemplazo de un concentrado comercial por ensilaje de contenido ruminal más sorgo en alimentación para pollos de engorde (Resumen). Rev MVZ Córdoba 2000; 5(2):34.

11. Adeniji $A A$, The feeding value of rumen content-maggot meal mixture in the diets of early weaned piglets. AJAVA 2008; 3(2):115-119.

12. Uicab LA, Sandoval CA. Uso del contenido ruminal y algunos residuos de la industria cárnica en la elaboración de composta. Tropical and Subtropical Agroecosystems 2003; 2:45-63.
13. Adams HR. Farmacología y terapéutica veterinaria. 2a ed. Zaragoza: Acribia; 2003.

14. Rios $\mathrm{PL}$, Rondón $\mathrm{MZ}$, Combellas JB y Álvarez ZR. Uso de morera (Morus sp.) y mata ratón (Gliricidia sepium) como sustitutos del alimento concentrado para corderos en crecimiento. Zootecnia Trop 2005; 23(1):49-60.

15. Andrew J, Niehaus DV. Rumenotomy. Vet Clin Noth Am Foof Anim Pract 2008; 24(2):341-347.

16. Cuesta Mazorra M, Cordero Herrera JF, Martin Hernández AR. Alternativa de la transfaunación en el tratamiento de rumiantes con insuficiente condición corporal. Engormix (En línea) 2007 Febrero (Fecha de acceso 25 de noviembre 2009); URL disponible en: http://www.engormix.com/alternativa_ transfaunacion_tratamiento_ rumiantes_s_articulos_1871_GDC.htm.

17. Roussel A. Procedimientos simples para casos complicados en la clínica de ganado vacuno: discusión sobre casos. Providesa (En línea) Mayo 2001 (Fecha de acceso 28 de noviembre de 2009); URL disponible en: http//www. prodivesa.com/simplmay.htm.

18. Quintero J, Barraza R, Lastra D. Obtención del líquido ruminal por tres diferentes técnicas en cavas lecheras y comparación de pH ruminal. [Trabajo de grado]. México: Universidad Nacional Autónoma de México, Facultad de Medicina Veterinaria y Zootecnia; 2000.

19. Cabrera Nuñes $A$, Rojas Mencio $P$, Renteria Iliana D, Serrano Solis A, Lopez Ortega M. Influencia de la suplementación sobre la ganancia de peso y calidad de la canal en borrego Dorper/Katahdin. Revista UDO Agricola 2007; 7(1):245-251.

20. Martinez Melendez I, Mena Hernandez I y Avalos Duran J. Granos y energéticos para los rumiantes. España: Corrua; 2001. 
21. Lara PGF, Olín MRO, Gómez GAV, Osorio AJ. Evaluacion de dos sistemas de alimentacion en ovinos para abasto en la zona del minicipio de xalatlaco, mexico. Asociación Mexicana de Médicos Veterinarios Especialistas en Bovinos A. C. (En línea) 2003 Junio (fecha de acceso 26 de abril de 2010); URL disponible en: http://ammveb. net/XXX\%20CNB/memorias $\% 20$ 2006/pequenos_rumiantes/carteles/ rpeq04.htm.

22. Álvarez G, Melgarejo L y Castañeda G. Ganancia de peso, conversión y eficacia alimentaria en ovinos alimentados con fruto (semilla con vaina) de parota (Entorolobium cyclocarpu) y pollinaza. Vet Mex 2003; 34(1):39-46.

23. García D, Cuesta M, Pedroso R. Administración de sulfato de cobre sobre la hemoquímica, hematología y bioactividad del líquido ruminal en vacas. Rev MVZ Córdoba 2005; 10(2):639-647.

24. Cano GN, Martínez OY. Enfermedades metabólicas de los animales domésticos. 2a Ed. Sucre, Colombia: Universidad de Sucre; 2000.

25. Ramos JJ, Cebrián LM, Contreras JS. Exploración del aparato digestivo: procedimientos primarios. Bovis 2000; (96):49-66.
26. García D, Cuesta M. Bioactividad del líquido ruminal y la salud-producción del ganado bovino. En: III Simposio de Medicina Veterinaria y Zootecnia. UCLV; Santa Clara Cuba: Memorias del III Simposio de Medicina Veterinaria y Zootecnia; 2005.

27. Roberts J, Delgado A. Enfermedades metabólicas y técnicas quirúrgicas. Acidosis ruminal subclínica: Diagnóstico por Ruminocentesis. Rev Investig Vet 2001; 12(2):215-221.

28. Montejo Cuenca E, Blanch Cuevas O, Pérez Freeman F, Martínez Vero O, Castañeda Sánchez M, Duvergel Rosseaux J, Sosa Tamayo W. Efecto del agua de cenizas sobre el estado ácido básico en una vaquería. REDVET Rev Electrón Vet (en línea) 2008 Julio (fecha de acceso 26 de abril de 2010) ; IX (7). URL disponible en: http://www.veterinaria.org/revistas/ redvet/n070708/070803.pdf.

29. Bramley E, Lean IJ, Fulkerson WJ, Stevenson MA, Rabiee AR, Costa ND. The definition of acidosis in dairy herds predominantly fed on pasture and concentrates. J Dairy Sci 2008; 91:308321. 